\title{
Drug-induced reductions in brain amyloid- $\beta$ levels may adversely affect cognition and behavior by a disruption of functional connectivity homeostasis
}

\author{
Bruno P Imbimbo' \& Nunzio Pomara*,2 \\ ${ }^{1}$ Research \& Development Department, Chiesi Farmaceutici, Largo Francesco Belloli 11/a, 43122 Parma, Italy \\ ${ }^{2}$ Department of Geriatric Psychiatry, Nathan Kline Institute, 140 Old Orangeburg Rd, Bldg 35, Orangeburg, NY 10962-1157, USA \\ *Author for correspondence: nunzio.pomara@NKI.rfmh.org
}

\author{
"Significant reductions in brain amyloid levels using other drugs, such as anti-A $\boldsymbol{\beta}$ \\ immunotherapies, have also been associated with cognitive decline and increase in \\ neuropsychiatric symptoms"
}

First draft submitted: 4 July 2019; Accepted for publication: 8 July 2019; Published online: 24 July 2019

Keywords: Alzheimer's disease $\bullet$ BACE1 inhibitors • resting state functional connectivity $\bullet$ verubecestat

Two recent publications described cognitive decline in clinical trials with the BACE1 inhibitor verubecestat in prodromal Alzheimer's disease (AD) [1] and atabecestat in unimpaired older individuals at risk of developing AD [2]. Both populations had elevated brain amyloid $(A \beta)$. In the case of verubecestat, the cognitive decline emerged in spite of successful target engagement, namely, significant reductions in brain burden and in soluble A $\beta$ species including CSF A 342 . Both BACE1 inhibitors were also associated with an increase in neuropsychiatric symptoms. In the case of verubecestat significant increases in frequency of psychiatric symptoms were observed in the highest verubecetsat dose group (40 mg once-a-day) compared with placebo: $10.3 \%$ versus 5.2\% for depression; $9.1 \%$ versus $4.3 \%$ for anxiety; $9.1 \%$ versus $4.5 \%$ on sleep disturbances; $5.6 \%$ versus $2.3 \%$ on psychotic symptoms and $9.3 \%$ versus $6.3 \%$ on suicidal ideation. The decline of the mean Neuropsychiatric Inventory score was also dose-dependent and reached statistical significance in the 40-mg dose group compared with placebo. The underlying mechanisms for the concomitant decline of cognitive and neuropsychiatric symptoms during treatment with BACE1 inhibitors was not discussed in any of these papers. It cannot simply be explained by a lack of validity of the $A \beta$ cascade hypothesis of $\mathrm{AD}$ as the latter should have resulted in just a lack of efficacy. Increased sensitivity to the adverse effects of excessive BACE1 inhibition on normal synaptic functions was suggested as a possible mechanism for the cognitive decline which was observed during the verubecestat trial [1]. Interestingly, verubecestat caused the onset of psychiatric symptoms also in cognitively normal elderly volunteers [3]. In a 4-week double-blind placebocontrolled study, 16 out of 60 healthy elderly subjects $(26.7 \%)$ treated with verubecestat (30-120 mg once-a-day) complained of psychiatric adverse events versus 0 out of 20 subjects on placebo $(\mathrm{p}=0.008)$. In experimental animals, the effects of $B A C E 1$ gene ablation on cognitive functions are complex. In transgenic mouse models of $\mathrm{AD}$ overexpressing human mutated forms of APP or PS, knocking the BACE1 gene attenuates or abolish memory deficit [4]. Similarly, a BACE1 inhibitor (NB-360) reversed neuronal hyperactivity and impaired long-range circuit function and attenuated memory deficit in APP23xPS45 transgenic mice overexpressing mutant human $A P P$ and PS1 [5]. On the other hand, transgenic $B A C E 1^{-/-}$normal mice revealed impairment in both spatial and working memories and decreased anxiety [6]. These data suggest that BACE1 is required for some normal hippocampal memory processes [7] and emotional function [8]. In cognitively normal elderly subjects, increased brain A $\beta$ burden has been associated with increased anxiety and depressive symptoms [9]. In subjects with mild cognitive impairment (MCI), $\mathrm{A} \beta$ burden of the brain is associated with an increased risk of having neuropsychiatric symptoms as compared to $\mathrm{MCI}$ without $\mathrm{A} \beta$ burden [10]. Thus, a reduction in brain $A \beta$ burden during verubecestat treatment should have resulted in a reduction rather than an increase in neuropsychiatric symptoms. Collectively these data suggest that 
alternative mechanisms should be considered for the cognitive decline and increase in neuropsychiatric symptoms associated with BACE1 inhibitor-induced brain and CSF A $\beta$ reductions.

Brain $A \beta$ plaques are associated with both hypo- and hyper-connectivity of functional networks as determined by resting state functional connectivity (RSFC), which may affect not only cognition [11], but also neuropsychiatric symptoms [12]. Studies in PDAPP and Tg2576 transgenic mice demonstrated that passive immunotherapy with $\mathrm{A} \beta$-targeting antibodies (3D6 and $\beta 1$ ) worsened abnormal neuronal hyperactivity and cognitive deficits [13]. On the other hand, treatment with the BACE1 inhibitor NB-360 normalized abnormal neuronal activity and attenuated memory deficit in APP23xPS45 transgenic mice [5]. These positive results in transgenic mouse models of $A D$ were not replicated with other BACE1 inhibitors like verubecestat, lanabecestat, elenbecestat, atabecestat and umibecestat [14]. Indeed, the cognitive decline which has been observed in clinical trials with these BACE1 inhibitors to date, suggest that normalization of neuronal activity may not be achieved in humans. We suggest that resting-state functional MRI and other techniques should be used to determine potential deleterious effects of drug-induced reduction in brain $A \beta$ on neuronal hyperactivity and RSFC in networks implicated in cognition [15]. Given the large body of data linking changes in neuronal activity and RSFC to neuropsychiatric symptoms [12], the proposed studies might also help to determine if a disruption of functional network homeostasis contributes to the increase in neuropsychiatric symptoms associated with BACE1 inhibitor treatment. Future studies should also determine if lower and slower drug-induced reductions of brain $A \beta$ plaques and other strategies, including primary prevention, can avert or attenuate the emergence of these adverse events and allow the detection of subtle disease-modifying effects. Significant reductions in brain amyloid levels using other drugs, such as anti-A $\beta$ immunotherapies, have also been associated with cognitive decline and increase in neuropsychiatric symptoms [14]. Therefore, it is important to determine if a disruption of brain functional connectivity associated with these treatments contributes to adverse cognitive and behavioral effects.

Financial \& competing interests disclosure

The authors have no relevant affiliations or financial involvement with any organization or entity with a financial interest in or financial conflict with the subject matter or materials discussed in the manuscript. This includes employment, consultancies, honoraria, stock ownership or options, expert testimony, grants or patents received or pending, or royalties.

No writing assistance was utilized in the production of this manuscript.

\section{References}

1. Egan MF, Kost J, Voss T et al. Randomized trial of verubecestat for prodromal Alzheimer's disease. N. Engl. J. Med. 380(15), 1408-1420 (2019).

2. Henley D, Raghavan N, Sperling R, Aisen P, Raman R, Romano G. Preliminary results of a trial of atabecestat in preclinical Alzheimer's disease. N. Engl. J. Med. 380(15), 1483-1485 (2019).

3. Forman M, Palcza J, Tseng J et al. Safety, tolerability, and pharmacokinetics of the $\beta$-site amyloid precursor protein-cleaving enzyme 1 inhibitor verubecestat (MK-8931) in healthy elderly male and female subjects. Clin. Transl. Sci. doi: 10.1111/cts.12645 (2019) (Epub ahead of print).

4. Cole SL, Vassar R. The Alzheimer's disease-secretase enzyme, BACE1. Mol. Neurodegener. 2(1), 22 (2007).

5. Keskin AD, Kekuš M, Adelsberger $\mathrm{H}$ et al. BACE inhibition-dependent repair of Alzheimer's pathophysiology. Proc. Natl Acad. Sci. USA 114(32), 8631-8636 (2017).

6. Laird FM, Cai H, Savonenko AV et al. BACE1, a major determinant of selective vulnerability of the brain to amyloid- $\beta$ amyloidogenesis, is essential for cognitive, emotional, and synaptic functions. J Neuroscience 25(50), 11693-11709 (2005).

7. Vassar R. Editorial: implications for BACE1 inhibitor clinical trials: adult conditional BACE1 knockout mice exhibit axonal organization defects in the hippocampus. J. Prev. Alzheimers Dis. 6(2), 78-84 (2019).

8. Plucińska K, Crouch B, Koss D et al. Knock-in of human BACE1 cleaves murine APP and reiterates Alzheimer-like phenotypes. J. Neurosci. 34(32), 10710-10728 (2014).

9. Krell-Roesch J, Lowe VJ, Neureiter J et al. Depressive and anxiety symptoms and cortical amyloid deposition among cognitively normal elderly persons: the Mayo Clinic Study of Aging. Int. Psychogeriatr. 30(2), 245-251 (2018).

10. Krell-Roesch J, Vassilaki M, Mielke MM et al. Cortical $\beta$-amyloid burden, neuropsychiatric symptoms, and cognitive status: the Mayo Clinic Study of Aging. Transl. Psychiatry 9(1), 123 (2019).

11. Palmqvist S, Schöll M, Strandberg $\mathrm{O}$ et al. Earliest accumulation of $\beta$-amyloid occurs within the default-mode network and concurrently affects brain connectivity. Nat. Commun. 8(1), 1214 (2017). 
12. Baker JT, Dillon DG, Patrick LM et al. Functional connectomics of affective and psychotic pathology. Proc. Natl Acad. Sci. USA 116(18), 9050-9059 (2019).

13. Busche MA, Grienberger $C$, Keskin $A D$ et al. Decreased amyloid- $\beta$ and increased neuronal hyperactivity by immunotherapy in Alzheimer's models. Nat. Neurosci. 18(12), 1725-1727 (2015).

14. Panza F, Lozupone M, Logroscino G, Imbimbo BP. A critical appraisal of amyloid- $\beta$-targeting therapies for Alzheimer disease. Nat. Rev. Neurol. 15, 73-88 (2019).

15. Pomara $\mathrm{N}$, Bruno $\mathrm{D}$. Basal forebrain resting functional activity associated with brain amyloid- $\beta$ burden in $\varepsilon 4$ carriers and older women: possible implications for baseline cognition and response to amyloid- $\beta$-based preventive trials. Radiology 290(3), 850-851 (2019). 
